\title{
New physics facing LFU and LFV tests in B physics
}

\section{Nejc Košnik*}

Department of Physics, University of Ljubljana

and

Jožef Stefan Institute, Ljubljana

E-mail: nejc.kosnikeijs.si

Lepton flavor universality in the Standard Model and hints of its violation in neutral $\left(R_{K}\right)$ and charged currents $\left(R_{D^{(*)}}\right)$ in $B$ decays are presented. We discuss model independent features as well as several leptoquark scenarios which are well suited to fit current values of $R_{K}$ and/or $R_{D^{(*)}}$.

Fourth Annual Large Hadron Collider Physics

13-18 June 2016

Lund, Sweden

${ }^{*}$ Speaker. 


\section{Introduction}

Lepton flavor universality is present on the level of Standard Model (SM) gauge couplings and is broken by the Higgs Yukawa couplings. Due to tiny neutrino masses and experimental blindness to neutrino flavors the PMNS mixing matrix can be safely neglected in $e^{+} e^{-}$or hadron collider. Lepton flavor universality (LFU) ratios, constructed as ratios of process rates that differ only in charged lepton flavor, are particularly clean quantities. This statement holds both on the experimental front, where common systematics of the two processes cancels out to some extent, as well as on the theoretical side where parametric uncertainties (e.g., CKM elements) and/or hadronic parameter uncertainties also cancel. In some cases the SM prediction of the LFU ratio is entirely determined by the charged lepton masses taking part in the process. Consider, e.g., the leptonic decay width induced by a $W$-exchange

$$
\Gamma_{P\left(\bar{q}_{j} q_{i}\right) \rightarrow \ell v}=\frac{G_{F}^{2}\left|V_{i j}\right|^{2} f_{P}^{2} m_{P}}{8 \pi} m_{\ell}^{2}\left(1-\frac{m_{\ell}^{2}}{m_{P}^{2}}\right)^{2} .
$$

When LFU ratio is taken with the above expression, the dependence on the CKM element vanishes, as well as on the hadronic decay constant (this latter feature is specific to two-body decays) ${ }^{1}$. LFU ratios have been repeatedly tested in meson decays and the results in Tab. 1 show remarkeble agreement with SM expectations, where disparate charged lepton masses are the only source of LFU breaking. However, charged current processes are not the most suitable for catching potential

\begin{tabular}{|c||c|c|}
\hline & SM & Experiment \\
\hline$R_{e / \mu}^{\pi}=\Gamma_{\pi \rightarrow e v} / \Gamma_{\pi \rightarrow \mu v}$ & $1.2352(1) \times 10^{-4}$ & $1.2327(23) \times 10^{-4}$ \\
\hline$R_{e / \mu}^{K}=\Gamma_{K \rightarrow e v} / \Gamma_{K \rightarrow \mu \nu}$ & $2.477(1) \times 10^{-5}$ & $2.488(10) \times 10^{-5}$ \\
\hline$R_{\tau / \mu}^{K}=\Gamma_{\tau \rightarrow K v} / \Gamma_{K \rightarrow \mu \nu}$ & $1.1162(3) \times 10^{-2}$ & $1.101(16) \times 10^{-2}$ \\
\hline$R_{\tau / \mu}^{B}=\Gamma_{B \rightarrow \tau v} / \Gamma_{B \rightarrow \mu \nu}$ & 223 & $\gtrsim 100$ \\
\hline
\end{tabular}

Table 1: Comparison of SM predictions and measured values of LFU ratios. Experimental values from [2].

beyond the large SM effects. Less SM background is expected in neutral current processes which are predicted to be strongly suppressed in the absence of exotic NP effects.

\section{Neutral current LFU: $R_{K}$}

The $\mathrm{LHCb}$ experiment reported an interesting result on the $\mathrm{LFU} \mu / e$ ratio in $b \rightarrow s \ell^{+} \ell^{-}$process [3]:

$$
R_{K}=\left.\frac{\mathscr{B}\left(B \rightarrow K \mu^{+} \mu^{-}\right)}{\mathscr{B}\left(B \rightarrow K e^{+} e^{-}\right)}\right|_{q^{2} \in[1,6] \mathrm{GeV}^{2}}=0.745 \pm_{0.074}^{0.090} \pm 0.036 \quad(\mathrm{LHCb})
$$

about $2.6 \sigma$ lower than the SM prediction $R_{K}^{S M}=1.00(3)[4,5]$. The theoretical uncertainty is dominated by the electromagnetic radiative corrections [5]. Investigation of the $B \rightarrow K^{(*)} \mu \mu$ process reveals deviations from SM prediction in angular observable $P_{5}^{\prime}$ in $B \rightarrow K^{*} \mu \mu$ as well as lower than expected differential spectra in various decay modes $\left(B \rightarrow K^{*} \mu \mu, B \rightarrow K \mu \mu, B \rightarrow \phi \mu \mu\right)[6,7]$ which suggests that the measured $R_{K}$ could be due to NP contributions in muonic decay modes.

\footnotetext{
${ }^{1}$ The ratio receives small electromagnetic corrections [1]
} 
A sensible starting point in studies of low-energy phenomenology of any NP model with degrees of freedom heavier than the weak scale is the SM, complemented at the electroweak scale by mass-dimension 6 operators (SM-EFT) $[8,9,10]$. More convenient for the treatment of low energy neutral current processes is an effective Hamiltonian that is matched onto to the SM-EFT through renormalization group (RG) running due to the full SM group above the electroweak scale [10] and due to strong and electromagnetic RG effects below the electroweak scale [11, 12],

$$
\mathscr{L}_{\text {eff }}=\frac{4 G_{F}}{\sqrt{2}} V_{t b} V_{t s}^{*}\left[\sum_{i=1}^{6} C_{i} \mathscr{O}_{i}+\sum_{i=7,8,9,10, S, P}\left(C_{i} \mathscr{O}_{i}+C_{i}^{\prime} \mathscr{O}_{i}^{\prime}\right)\right] .
$$

Among all the operators we will list only the semileptonic ones, which carry a lepton index and may be responsible for lepton universality violation:

$$
\begin{aligned}
\mathscr{O}_{9}^{(\prime)} & =\frac{e^{2}}{(4 \pi)^{2}}\left(\bar{s} \gamma_{\mu} P_{L(R)} b\right)\left(\bar{\ell} \gamma^{\mu} \ell\right), & \mathscr{O}_{10}^{(\prime)} & =\frac{e^{2}}{(4 \pi)^{2}}\left(\bar{s} \gamma_{\mu} P_{L(R)} b\right)\left(\bar{\ell} \gamma^{\mu} \gamma_{5} \ell\right), \\
\mathscr{O}_{S}^{(\prime)} & =\frac{e^{2}}{(4 \pi)^{2}}\left(\bar{s} P_{R(L)} b\right)(\bar{\ell} \ell), & \mathscr{O}_{P}^{(\prime)} & =\frac{e^{2}}{(4 \pi)^{2}}\left(\bar{s} P_{R(L)} b\right)\left(\bar{\ell} \gamma_{5} \ell\right) .
\end{aligned}
$$

In the following we will only consider modification of the processes with muons in the final state and accordingly we set $\ell=\mu$ in the above operators, while the $b \rightarrow$ see interactions will be assumed SM-like. The values of the SM Wilson coefficients at scale $m_{B}$ are $C_{9}^{\mathrm{SM}}=-C_{10}^{\mathrm{SM}}=4.2$. Furthermore, the SM-EFT matching onto the low energy Hamiltonian for $b \rightarrow s \ell \ell$ processes already predicts that no tensor operators would be generated, and that scalar operators are related to pseudoscalars $[13,14,15,12], C_{S}=-C_{P}, C_{S}^{\prime}=C_{P}^{\prime}$. In the following $C_{i}$ will refer to the value of the Wilson coefficient relative to its $\mathrm{SM}$ value.

The LFU universality $R_{K}$ itself does not allow explanation in terms of scalar and pseudoscalar operators, $\mathscr{O}_{S}^{\left({ }^{\prime}\right)}$ and $\mathscr{O}_{P}^{(')}$, since their size needed would cause excessive $B_{s} \rightarrow \mu \mu$ branching fraction. One scenario that fits both $R_{K}$ and the rest of $b \rightarrow s \mu \mu$ data is the left-handed current scenario with $C_{9}=-C_{10}[16,17]$, however this is not the only possibility. Vector lepton currents $C_{9}, C_{9}^{\prime}$, induced by $Z^{\prime}$ coupled to muons and taus have been studied in $[18,19]$. Scenario with right-handed quark current, $C_{9}^{\prime}=-C_{10}^{\prime}$, can address $R_{K}$, and can be potentially resolved from the left-handed scenario in other LFU ratios [17, 20, 21].

The analysis of the $C_{9}^{\prime}=-C_{10}^{\prime}$ scenario was carried out in [21]. The experimental constraints that have been imposed are the semileptonic and leptonic decay rates:

$$
\begin{aligned}
\left.\mathscr{B}\left(B^{+} \rightarrow K^{+} \mu^{+} \mu^{-}\right)\right|_{q^{2} \in[15,22] \mathrm{GeV}^{2}} & =(8.5 \pm 0.3 \pm 0.4) \times 10^{-8} \\
\mathscr{B}\left(B_{s} \rightarrow \mu^{+} \mu^{-}\right) & =\left(2.8_{-0.6}^{+0.7}\right) \times 10^{-9}
\end{aligned}
$$

The hadronic form factors that were employed had been calculated in unquenched lattice QCD simulation [23], while for the $B_{s}$ decay constant the FLAG average [24] was used. Prediction of $R_{K}$ cancels out form factor errors and reads

$$
R_{K}\left(C_{10}^{\prime}\right)=1.001(1)-0.46 \operatorname{Re}\left[C_{10}^{\prime}\right]-0.094(3) \operatorname{Im}\left[C_{10}^{\prime}\right]+0.057(1)\left|C_{10}^{\prime}\right|^{2},
$$

where the remaining uncertainties are indicated by the numbers in parentheses. In Fig. 1 we show contours of constant $R_{K}$ in the $C_{10}^{\prime}$ plane using the formula (2.5). Mapping the fitted region (green) 
to $R_{K}$ we obtain the prediction

$$
R_{K}^{\text {pred. }}=0.88 \pm 0.08,
$$

which is indeed in good agreement with the LHCb measurement. Possible UV completion of

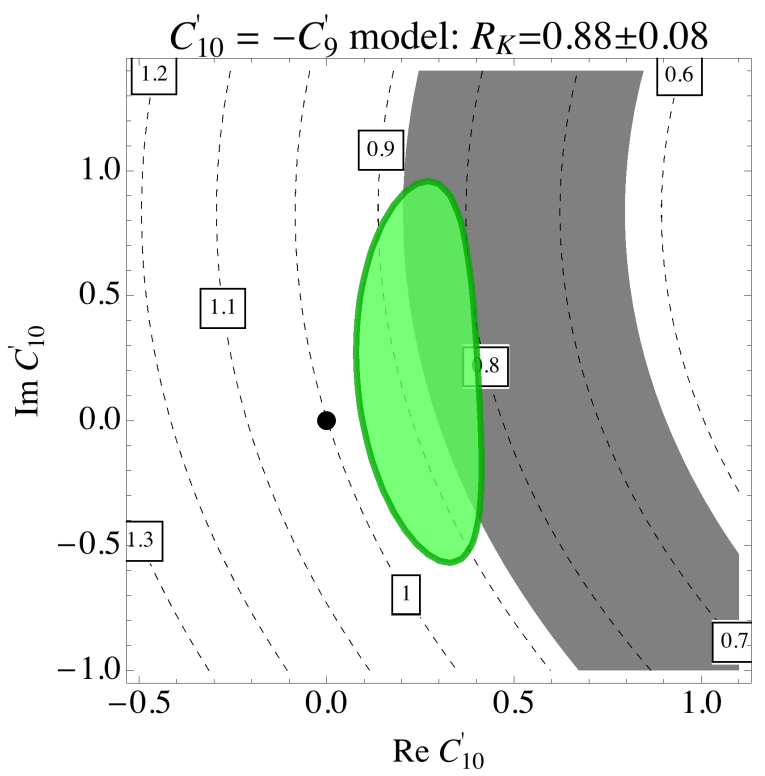

Figure 1: $R_{K}$ are denoted by dashed lines. Gray region represents the LHCb measurement of $R_{K}$. Green contour is the $1 \sigma$ fitted region to $\mathscr{B}\left(B_{s} \rightarrow \mu^{+} \mu^{-}\right)$and $\mathscr{B}\left(B \rightarrow K \mu^{+} \mu^{-}\right)$.

the $C_{9}^{\prime}=-C_{10}^{\prime}$ model is a scalar leptoquark $\Delta$ in the SM representation $(3,2,1 / 6)$. The defining Lagrangian contains a leptoquark (LQ) scalar field $\Delta$ that is coupled to the SM fermions in Yukawa terms:

$$
\begin{aligned}
\mathscr{L} & =Y_{i j} \bar{L}_{i} i \tau^{2} \Delta^{*} d_{R j}+\text { h.c. } \\
& =Y_{i j}\left(-\bar{\ell}_{L i} d_{R j} \Delta^{(2 / 3) *}+\bar{v}_{L k}\left(V^{\mathrm{PMNS}}\right)_{k i}^{\dagger} d_{R j} \Delta^{(-1 / 3) *}\right)+\text { h.c.. }
\end{aligned}
$$

At the scale $m_{B}$ the Wilson coefficients are expressed in terms of LQ Yukawas

$$
C_{10}^{\prime}=-C_{9}^{\prime}=\frac{\pi}{2 \sqrt{2} G_{F} V_{t b} V_{t s}^{*} \alpha} \frac{Y_{\mu b} Y_{\mu s}^{*}}{m_{\Delta}^{2}} .
$$

Mass of $\Delta$ from direct searches at the LHC should be larger than few 100's of GeV, with precise limit depending on its pattern of decay branching fractions. For indirect effects that we are interested in the precise mass is not an issue since it is the combination of Yukawa couplings and masses that enters predictions here and all amplitudes (with the exception of neutral meson mixing) scale as $\sim Y^{2} / M_{\Delta}^{2}$. From different scaling in meson $B_{s}-\bar{B}_{s}$ mixing we conclude that $\Delta$ should be lighter than $\sim 100 \mathrm{TeV}$ in order to stay in the perturbative regime. In [21] we have predicted, alongside $R_{K}$, slightly enhanced $B \rightarrow K^{(*)} v v$ decay mode and enhanced LFU ratio $R_{K^{*}}=1.11(8)$.

Further LQ scenarios have been proposed in the recent literature. A weak triplet state $(\overline{3}, 3,1 / 3)$ that implements $C_{9}=-C_{10}$ effective theory has been presented in [17]. Weak singlet state $(\overline{3}, 1,1 / 3)$ was proposed in [25] to explain $R_{K}$ and alongside with it also the charged LFU puzzle, $R_{D^{(*)}}$, to 
be discussed in the next section. Both LQ states with hypercharge $1 / 3$ have additional diquark couplings which must be suppressed to maintain stability of the proton. Furthermore, viability of $(\overline{3}, 1,1 / 3)$ scenario due to additional flavor constraints has been questioned in [26]. A vector leptoquark scenario in representation $(3,3,2 / 3)$ that addresses $R_{K}$ and $R_{D^{(*)}}$ will be presented below.

\section{Charged current LFU: $R_{D^{(*)}}$}

In the charged-current induced semileptonic $B$ decays we are witnessing persistent indications of disagreement with the SM prediction of lepton flavor universality (LFU) ratio in the $\tau / \mu$ and/or $\tau / e$ sector. Namely, in the ratio $R_{D^{(*)}}=\frac{\Gamma\left(B \rightarrow D^{(*)} \tau^{-} \bar{v}\right)}{\Gamma\left(B \rightarrow D^{(*)} \ell^{-} \bar{v}\right)}$ the deviation from the SM is at $4 \sigma$ level [27, 28]. The significance of the discrepancy is driven by the experimental world average [27],

$$
R_{D}^{\text {exp }}=0.397 \pm 0.040 \pm 0.028, \quad R_{D^{*}}^{\exp }=0.316 \pm 0.016 \pm 0.010
$$

with a correlation coefficient of -0.21 . The SM predictions are much more precise:

$$
R_{D}^{\mathrm{SM}}=0.297 \pm 0.017, \quad R_{D^{*}}^{\mathrm{SM}}=0.252 \pm 0.003
$$

and have been obtained in [29] and [30], respectively. The $R_{D^{(*)}}$ puzzle has attracted a lot of attention recently (see e.g., [31, 32, 33, 34, 35]).

In terms of the effective theory framework the vector $\left(g_{V}\right)$, scalar $\left(g_{S}\right)$, and tensor $\left(g_{T}\right)$ operators modifying the semi-tauonic decays give best fits to the measured $R_{D^{(*)}}[36,37]$ :

$$
\mathscr{L}=-\frac{4 G_{F} V_{c b}}{\sqrt{2}}\left[\left(1+g_{V}\right)\left(\bar{\tau}_{L} \gamma^{\mu} v_{L}\right)\left(\bar{c}_{L} \gamma_{\mu} b_{L}\right)+g_{S}\left(\bar{\tau}_{R} v_{L}\right)\left(\bar{c}_{R} b_{L}\right)+g_{T}\left(\bar{\tau}_{R} \sigma^{\mu v} v_{L}\right)\left(\bar{c}_{R} \sigma_{\mu v} b_{L}\right)\right] .
$$

Since the measured LFU violation requires large enhancement of the SM tree-level process it is compulsory to invoke a NP model contributing at tree-level. For NP studies involving charged colorless scalars see, e.g., [38, 39, 40, 41], whereas colored states (leptoquarks) have been pursued in, e.g., $[42,43,25,44]$.

First, consider a model with a scalar leptoquark $R_{2}$ in the representation $(3,2,7 / 6)$ that couples non-chirally

$$
\mathscr{L}_{\mathrm{LQ}}=\bar{\ell}_{R} Y R_{2}^{\dagger} Q+\bar{u}_{R} Z i \tau_{2} R_{2}^{T} L+\text { h.c.. }
$$

Here we have chosen $Y$ and $Z$ Yukawa matrices in a way to have minimal number of non-zero elements and still allow explaining $R_{D^{(*)}}$. $Y$ only couples $\tau_{R}$ to $b_{L}, Z$ couples $c_{R}$ to all neutrinos and charged leptons. It has been shown in a realistic $S U(5)$ unification model [42] that $\mu \rightarrow e \gamma$ is the most relevant constraint that forces $Y_{\tau b}$ to be large and consequently $R_{2}$ is well suited to direct searches in $\tau b$ final states. Left panel in Fig. 2 outlines the effect of various constraints.

The second scenario is the one with the vector $\mathrm{LQ}, U_{3}(3,3,2 / 3)$. Such weak triplet always contributes to left-handed current operators, both in charged and neutral currents, and in can turn interfere with the SM contributions very effectively to address $R_{D^{(*)}}$ as well as $R_{K}$ [45]:

$$
\begin{aligned}
\mathscr{L}_{U_{3}}= & U_{3 \mu}^{(2 / 3)}\left[(V g)_{i j} \bar{u}_{i} \gamma^{\mu} P_{L} v_{j}-g_{i j} \bar{d}_{i} \gamma^{\mu} P_{L} \ell_{j}\right] \\
& +U_{3 \mu}^{(5 / 3)}(\sqrt{2} V g)_{i j} \bar{u}_{i} \gamma^{\mu} P_{L} \ell_{j} \\
& +U_{3 \mu}^{(-1 / 3)}(\sqrt{2} g)_{i j} \bar{d}_{i} \gamma^{\mu} P_{L} v_{j}+\text { h.c.. }
\end{aligned}
$$



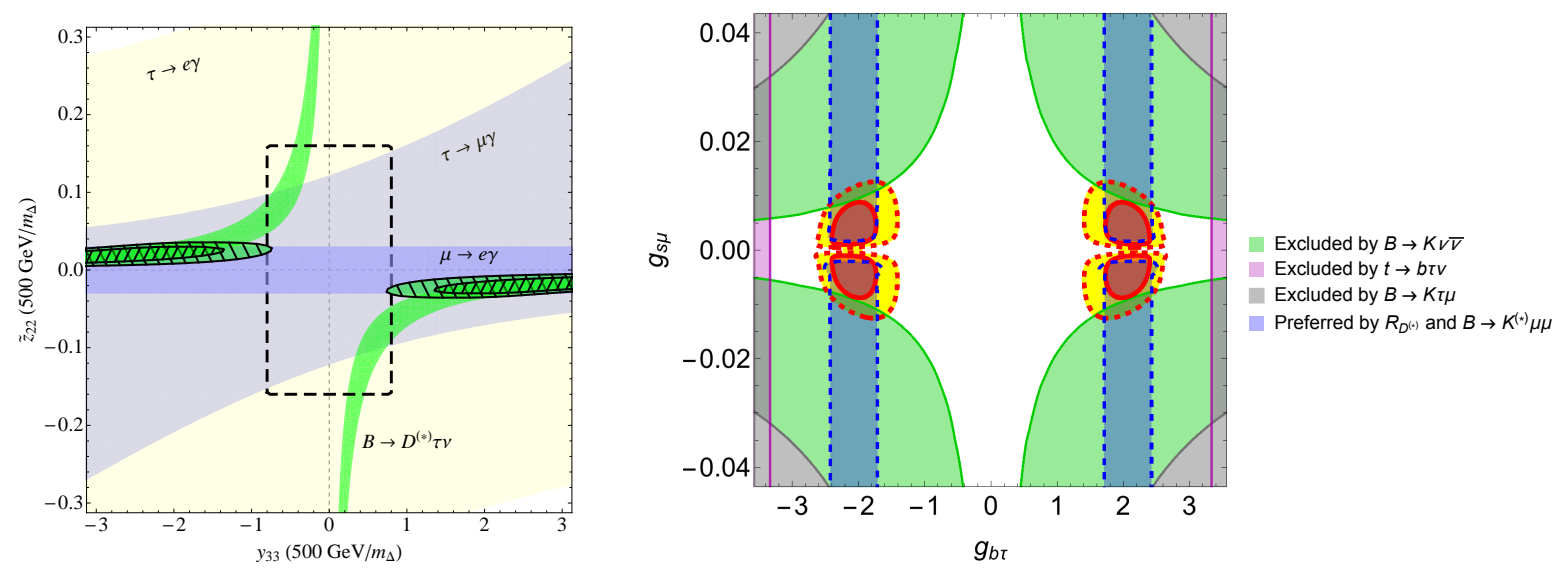

Figure 2: Left panel: Constraints on the $R_{2}$ leptoquark scenario embedded in $S U(5)$ GUT theory [42]. Dashed frame region ensures perturbativity of the Yukawa couplings all the way to the GUT scale. Hatched green area denotes the best fit region. Right panel: Constraints on the vector LQ $U_{3}$, as discussed in [45]. Red and yellow regions are the $1-$ and $2-\sigma$ fitted regions.

Couplings are judiciously chosen in the down quark-charged lepton sector:

$$
g=\left(\begin{array}{ccc}
0 & 0 & 0 \\
0 & g_{s \mu} & 0 \\
0 & g_{b \mu} & g_{b \tau}
\end{array}\right), \quad V g=\left(\begin{array}{ccc}
0 & V_{u s} g_{s \mu}+V_{u b} g_{b \mu} & V_{u b} g_{b \tau} \\
0 & V_{c s} g_{s \mu}+V_{c b} g_{b \mu} & V_{c b} g_{b \tau} \\
0 & V_{t s} g_{s \mu}+V_{t b} g_{b \mu} & V_{t b} g_{b \tau}
\end{array}\right),
$$

and are related to up-quarks and neutrino interactions by CKM rotation.

Again, $R_{D^{(*)}}$ requires large coupling to the third generation matter. For this scenario it is the $B \rightarrow K v v$ constraint that most effectively probes the LFV couplings that also drive the $B \rightarrow K \tau \mu$, as seen in right-panel in Fig. 2.

\section{Conclusion}

The two recent LFU violating observables can be explained by tree-level LQ contributions. We have demonstrated that a scalar leptoquark $\Delta(3,2,1 / 6)$ can successfully explain $R_{K}$ whereas $R_{2}(3,2,7 / 6)$ can shift $R_{D^{(*)}}$ closer to experiment. Finally, triplet vector leptoquark $U_{3}(3,3,2 / 3)$ can modify the left-handed current operators and explain both LFU puzzles. In all the above cases we observe that explaining $R_{D^{(*)}}$ calls for large coupling between $\tau$ and $b$. Lepton flavor violation constraints generally do not allow for additional sizable LQ couplings to fermions. In all the considered scenarios bounds on the LFV processes provide the most stringent constraint, e.g. $\mu \rightarrow$ $e \gamma$ for $R_{2}(3,2,7 / 6)$ and $B \rightarrow K v v$ for $U_{3}(3,3,2 / 3)$.

\section{References}

[1] V. Cirigliano and I. Rosell, Phys. Rev. Lett. 99, 231801 (2007) doi:10.1103/PhysRevLett.99.231801 [arXiv:0707.3439 [hep-ph]].

[2] K. A. Olive et al. [Particle Data Group Collaboration], Chin. Phys. C 38, 090001 (2014). doi:10.1088/1674-1137/38/9/090001 
[3] R. Aaij et al. [LHCb Collaboration], Phys. Rev. Lett. 113, 151601 (2014) doi:10.1103/PhysRevLett.113.151601 [arXiv:1406.6482 [hep-ex]].

[4] G. Hiller and F. Kruger, Phys. Rev. D 69, 074020 (2004) doi:10.1103/PhysRevD.69.074020 [hep-ph/0310219].

[5] M. Bordone, G. Isidori and A. Pattori, Eur. Phys. J. C 76, no. 8, 440 (2016) doi:10.1140/epjc/s10052-016-4274-7 [arXiv:1605.07633 [hep-ph]].

[6] R. Aaij et al. [LHCb Collaboration], JHEP 1406, 133 (2014) doi:10.1007/JHEP06(2014)133 [arXiv:1403.8044 [hep-ex]].

[7] R. Aaij et al. [LHCb Collaboration], JHEP 1509, 179 (2015) doi:10.1007/JHEP09(2015)179 [arXiv:1506.08777 [hep-ex]].

[8] W. Buchmuller and D. Wyler, Nucl. Phys. B 268, 621 (1986). doi:10.1016/0550-3213(86)90262-2

[9] B. Grzadkowski, M. Iskrzynski, M. Misiak and J. Rosiek, JHEP 1010, 085 (2010) doi:10.1007/JHEP10(2010)085 [arXiv:1008.4884 [hep-ph]].

[10] R. Alonso, E. E. Jenkins, A. V. Manohar and M. Trott, JHEP 1404, 159 (2014) doi:10.1007/JHEP04(2014)159 [arXiv:1312.2014 [hep-ph]].

[11] G. Buchalla, A. J. Buras and M. E. Lautenbacher, Rev. Mod. Phys. 68, 1125 (1996) doi:10.1103/RevModPhys.68.1125 [hep-ph/9512380].

[12] F. Feruglio, P. Paradisi and A. Pattori, arXiv:1606.00524 [hep-ph].

[13] R. Alonso, B. Grinstein and J. Martin Camalich, Phys. Rev. Lett. 113, 241802 (2014) doi:10.1103/PhysRevLett.113.241802 [arXiv:1407.7044 [hep-ph]].

[14] R. Alonso, B. Grinstein and J. Martin Camalich, JHEP 1510, 184 (2015) doi:10.1007/JHEP10(2015)184 [arXiv:1505.05164 [hep-ph]].

[15] O. Catà and M. Jung, Phys. Rev. D 92, no. 5, 055018 (2015) doi:10.1103/PhysRevD.92.055018 [arXiv:1505.05804 [hep-ph]].

[16] G. Hiller and M. Schmaltz, Phys. Rev. D 90, 054014 (2014) doi:10.1103/PhysRevD.90.054014 [arXiv:1408.1627 [hep-ph]].

[17] G. Hiller and M. Schmaltz, JHEP 1502, 055 (2015) doi:10.1007/JHEP02(2015)055 [arXiv:1411.4773 [hep-ph]].

[18] W. Altmannshofer, S. Gori, M. Pospelov and I. Yavin, Phys. Rev. D 89, 095033 (2014) doi:10.1103/PhysRevD.89.095033 [arXiv:1403.1269 [hep-ph]].

[19] W. Altmannshofer and I. Yavin, Phys. Rev. D 92, no. 7, 075022 (2015) doi:10.1103/PhysRevD.92.075022 [arXiv:1508.07009 [hep-ph]].

[20] W. Altmannshofer and D. M. Straub, Eur. Phys. J. C 73, 2646 (2013) doi:10.1140/epjc/s10052-013-2646-9 [arXiv:1308.1501 [hep-ph]].

[21] D. Bečirević, S. Fajfer and N. Košnik, Phys. Rev. D 92, no. 1, 014016 (2015) doi:10.1103/PhysRevD.92.014016 [arXiv:1503.09024 [hep-ph]].

[22] V. Khachatryan et al. [CMS and LHCb Collaborations], Nature 522, 68 (2015) doi:10.1038/nature14474 [arXiv:1411.4413 [hep-ex]]. 
[23] C. Bouchard et al. [HPQCD Collaboration], Phys. Rev. D 88, no. 5, 054509 (2013) Erratum: [Phys. Rev. D 88, no. 7, 079901 (2013)] doi:10.1103/PhysRevD.88.079901, 10.1103/PhysRevD.88.054509 [arXiv:1306.2384 [hep-lat]].

[24] S. Aoki et al., Eur. Phys. J. C 74, 2890 (2014) doi:10.1140/epjc/s10052-014-2890-7 [arXiv:1310.8555 [hep-lat]].

[25] M. Bauer and M. Neubert, Phys. Rev. Lett. 116, no. 14, 141802 (2016) doi:10.1103/PhysRevLett.116.141802 [arXiv:1511.01900 [hep-ph]].

[26] D. Bečirević, N. Košnik, O. Sumensari and R. Zukanovich Funchal, arXiv:1608.07583 [hep-ph].

[27] Y. Amhis et al. [Heavy Flavor Averaging Group (HFAG) Collaboration], arXiv:1412.7515 [hep-ex].

[28] T. Blake, G. Lanfranchi and D. M. Straub, arXiv:1606.00916 [hep-ph].

[29] H. Na et al. [HPQCD Collaboration], Phys. Rev. D 92, no. 5, 054510 (2015) Erratum: [Phys. Rev. D 93, no. 11, 119906 (2016)] doi:10.1103/PhysRevD.93.119906, 10.1103/PhysRevD.92.054510 [arXiv:1505.03925 [hep-lat]].

[30] S. Fajfer, J. F. Kamenik and I. Nisandzic, Phys. Rev. D 85, 094025 (2012) doi:10.1103/PhysRevD.85.094025 [arXiv:1203.2654 [hep-ph]].

[31] A. Crivellin and S. Pokorski, Phys. Rev. Lett. 114, no. 1, 011802 (2015) doi:10.1103/PhysRevLett.114.011802 [arXiv:1407.1320 [hep-ph]].

[32] B. Bhattacharya, A. Datta, D. London and S. Shivashankara, Phys. Lett. B 742, 370 (2015) doi:10.1016/j.physletb.2015.02.011 [arXiv:1412.7164 [hep-ph]].

[33] S. Bhattacharya, S. Nandi and S. K. Patra, Phys. Rev. D 93, no. 3, 034011 (2016) doi:10.1103/PhysRevD.93.034011 [arXiv:1509.07259 [hep-ph]].

[34] C. Hati, G. Kumar and N. Mahajan, JHEP 1601, 117 (2016) doi:10.1007/JHEP01(2016)117 [arXiv: 1511.03290 [hep-ph]].

[35] Y. Sakaki, M. Tanaka, A. Tayduganov and R. Watanabe, Phys. Rev. D 91, no. 11, 114028 (2015) doi:10.1103/PhysRevD.91.114028 [arXiv:1412.3761 [hep-ph]].

[36] D. Bečirević, N. Košnik and A. Tayduganov, Phys. Lett. B 716, 208 (2012) doi:10.1016/j.physletb.2012.08.016 [arXiv:1206.4977 [hep-ph]].

[37] M. Freytsis, Z. Ligeti and J. T. Ruderman, Phys. Rev. D 92, no. 5, 054018 (2015) doi:10.1103/PhysRevD.92.054018 [arXiv:1506.08896 [hep-ph]].

[38] A. Crivellin, C. Greub and A. Kokulu, Phys. Rev. D 86, 054014 (2012) doi:10.1103/PhysRevD.86.054014 [arXiv:1206.2634 [hep-ph]].

[39] A. Celis, M. Jung, X. Q. Li and A. Pich, JHEP 1301, 054 (2013) doi:10.1007/JHEP01(2013)054 [arXiv:1210.8443 [hep-ph]].

[40] P. Ko, Y. Omura and C. Yu, JHEP 1303, 151 (2013) doi:10.1007/JHEP03(2013)151 [arXiv:1212.4607 [hep-ph]].

[41] A. Crivellin, J. Heeck and P. Stoffer, Phys. Rev. Lett. 116, no. 8, 081801 (2016) doi:10.1103/PhysRevLett.116.081801 [arXiv:1507.07567 [hep-ph]].

[42] I. Doršner, S. Fajfer, N. Košnik and I. Nišandžić, JHEP 1311, 084 (2013) doi:10.1007/JHEP11(2013)084 [arXiv:1306.6493 [hep-ph]]. 
[43] Y. Sakaki, M. Tanaka, A. Tayduganov and R. Watanabe, Phys. Rev. D 88, no. 9, 094012 (2013) doi:10.1103/PhysRevD.88.094012 [arXiv:1309.0301 [hep-ph]].

[44] X. Q. Li, Y. D. Yang and X. Zhang, JHEP 1608, 054 (2016) doi:10.1007/JHEP08(2016)054 [arXiv:1605.09308 [hep-ph]].

[45] S. Fajfer and N. Košnik, Phys. Lett. B 755, 270 (2016) doi:10.1016/j.physletb.2016.02.018 [arXiv:1511.06024 [hep-ph]]. 